\title{
Weak KAM Solutions of a Discrete-Time Hamilton-Jacobi Equation in a Minimax Framework
}

\author{
Porfirio Toledo \\ Facultad de Matemáticas, UV Zona Universitaria, 91090 Xalapa, Ver, Mexico \\ Correspondence should be addressed to Porfirio Toledo; ptoledo@uv.mx
}

Received 28 January 2013; Accepted 31 May 2013

Academic Editor: Stepan Agop Tersian

Copyright (C) 2013 Porfirio Toledo. This is an open access article distributed under the Creative Commons Attribution License, which permits unrestricted use, distribution, and reproduction in any medium, provided the original work is properly cited.

\begin{abstract}
The purpose of this paper is to study the existence of solutions of a Hamilton-Jacobi equation in a minimax discrete-time case and to show different characterizations for a real number called the critical value, which plays a central role in this work. We study the behavior of solutions of this problem using tools of game theory to obtain a "fixed point" of the Lax operator associated, considering some facts of weak KAM theory to interpret these solutions as discrete viscosity solutions. These solutions represent the optimal payoff of a zero-sum game of two players, with increasingly long time payoffs. The developed techniques allow us to study the behavior of an infinite time game without using discount factors or average actions.
\end{abstract}

\section{Introduction}

The Hamilton-Jacobi equation is a very important tool for the study of Lagrangian systems and control theory. In the case of convex Lagrangians, Fathi, Mather, and Mañe have perfected several techniques to understand the solutions of this equation ([1-7]). These techniques can be translated to a discrete case where, instead of a Lagrangian, there is a function $V(x, y)$ of two variables in the configuration space.

The aim of this paper is to study a discrete-time system that emulates a convex and concave Lagrangian. We need to study the behavior of solutions of a discrete-time version of a Hamilton-Jacobi equation in the context of game theory. A real number, called the critical value, plays a central role in this work; this number is the asymptotic average action of optimal trajectories. We study the existence and characterization of solutions of a Hamilton-Jacobi equation for this kind of game. A relevant aspect is that these techniques allow us to study infinite-time games without using discount factors or average actions.

Consider the compact metric spaces $M$ and $N$, which are the state spaces for players $J_{M}$ and $J_{N}$, respectively. For $(x, y) \in M \times N$, a continuous function $g: M \times N \rightarrow \mathbb{R}$, and a Lipschitz function $V: M \times N \times M \times N \rightarrow \mathbb{R}$, we define the static game payoff by

$$
P_{g}\left(x_{1}, y_{1}\right):=g\left(x_{1}, y_{1}\right)-V\left(x, y, x_{1}, y_{1}\right) .
$$

The function $P$ is the amount that player $J_{M}$ obtains from $J_{N}$ and vice versa; so player $J_{M}$ wants to maximize the payoff $P$, while player $J_{N}$ wants to minimize it. The lower game value is defined by

$$
\mathscr{L}^{1} g(x, y):=\sup _{x_{1} \in M} \inf _{y_{1} \in N} P_{g}\left(x_{1}, y_{1}\right) .
$$

It is observed in [8] that it is possible to express the lower game value as follows:

$$
\mathscr{L}^{1} g(x, y)=\inf _{\theta(z)} \sup _{z \in M} P_{g}(z, \theta(z)),
$$

where the function $\theta: M \rightarrow N$ is called a strategy for player $J_{N}$. These functions are very important in this work and will be defined in general for a zero-sum game with finite horizon.

For $n \in \mathbb{Z}^{+}$, consider the finite horizon problem, where players $J_{M}$ and $J_{N}$ alternate their moves beginning at initial state $\left(x_{0}, y_{0}\right)$ and setting with them the state trajectories 
determined by finite sequences $\bar{x}(n) \subset M$ and $\bar{y}(n) \subset$ $N$, denoted by $\bar{x}(n):=\left\{x_{0}, x_{1}, \ldots, x_{n}\right\}$ and $\bar{y}(n):=\left\{y_{0}\right.$, $\left.y_{1}, \ldots, y_{n}\right\}$, respectively. The state of the system depends on the initial condition $\left(x_{0}, y_{0}\right)$ and the players decisions to get from $\left(x_{i}, y_{i}\right)$ to $\left(x_{i+1}, y_{i+1}\right)$, where $i=0,1, \ldots, n-1$. For $(x, y) \in M \times N$, we will denote by

$$
\begin{aligned}
& S_{M}(x, n):=\left\{\bar{x}(n)=\left\{x_{i}\right\}_{i=0}^{n}: x_{0}=x\right\}, \\
& S_{N}(y, n):=\left\{\bar{y}(n)=\left\{y_{i}\right\}_{i=0}^{n}: y_{0}=y\right\},
\end{aligned}
$$

the sets of finite sequences in $M$ and $N$, with initial states $x \in$ $M$ and $y \in N$. We define the action by

$$
A^{n}(\bar{x}(n), \bar{y}(n)):=\sum_{i=0}^{n-1} V\left(x_{i}, y_{i}, x_{i+1}, y_{i+1}\right),
$$

and the game payoff by

$$
P_{g}(\bar{x}(n), \bar{y}(n)):=g\left(x_{n}, y_{n}\right)-A^{n}(\bar{x}(n), \bar{y}(n)) .
$$

Then, the lower game value with finite horizon $n$ and initial state $(x, y) \in M \times N$ is

$$
\begin{aligned}
& \mathscr{L}^{n} g(x, y) \\
& \quad:=\sup _{x_{n} \in M} \inf _{y_{n} \in N} \sup _{x_{n-1} \in M} \inf _{y_{n-1} \in N} \ldots \sup _{x_{1} \in M} \inf _{y_{1} \in N} P_{g}(\bar{x}(n), \bar{y}(n)) .
\end{aligned}
$$

However, $\mathscr{L}^{n}$ can be defined directly using progressive strategies (see [8-10]). The idea is to use a tool that allows player $J_{N}$ to select one point $y_{i}$ knowing the current and past opponent choices, without knowing the future choices. To this end, we define a progressive strategy $\Theta(n)$ for player $J_{N}$ as a function $\Theta(n): S_{M}(x, n) \rightarrow S_{N}(y, n)$ which satisfies the following property: for each $m \in \mathbb{Z}$ such that $0 \leq m \leq n$ and $\bar{x}(n), \bar{z}(n) \in S_{M}(x, n)$, we have

$$
\begin{aligned}
x_{i} & =z_{i}, \quad 0 \leq i \leq m \\
& \Longrightarrow \Theta(n)[\bar{x}(n)]_{i}=\Theta(n)[\bar{z}(n)]_{i}, \quad 0 \leq i \leq m,
\end{aligned}
$$

where $\Theta(n)[\bar{x}(n)]_{i}$ is the $i$ th element of sequence $\Theta(n)[\bar{x}(n)] \epsilon$ $S_{N}(y, n)$. The set of progressive strategies for player $J_{N}$ is denoted by

$$
\begin{aligned}
\mathscr{E}_{N}(x, y, n) & \\
:= & \left\{\Theta(n): S_{M}(x, n)\right. \\
& \left.\longrightarrow S_{N}(y, n) \text { progressive strategy }\right\} .
\end{aligned}
$$

Finally, the lower game value can be expressed as

$$
\mathscr{L}^{n} g(x, y)=\inf _{\Theta(n)} \sup _{\bar{x}(n)} P_{g}(\bar{x}(n), \Theta(n)[\bar{x}(n)]),
$$

where $\bar{x}(n) \in S_{M}(x, n)$ and $\Theta(n) \in \mathscr{E}_{N}(x, y, n)$. This function is called Lax operator and will be essential for understanding the asymptotic behavior of game payoffs. The Proposition 6 shows that

$$
\mathscr{L}^{n} g(x, y)=\mathscr{L}_{n \text { times }}^{1} \circ \ldots \circ \mathscr{L}^{1} g(x, y) .
$$

In the next section of this paper, the objective is to prove the existence of a kind of "fixed point" of the Lax operator. We will adapt the methods developed in the work of Fathi ([3]) about weak KAM theory, now in a minimax context of game theory. It will be necessary to prove that the Lax operator $\mathscr{L}^{n}$, defined as in (10), satisfies some important properties: the semigroup property that is also call Dynamic Programming Principle (see [8-10]), a regularity property, monotony, and weak contraction.

Theorem 1. If $M$ and $N$ are compact metric spaces and $V$ : $M \times N \times M \times N \rightarrow \mathbb{R}$ is a Lipschitz function, then there exist a Lipschitz function $u \in C(M \times N, \mathbb{R})$ and a unique constant $c \in \mathbb{R}$, such that

$$
\mathscr{L}^{n} u=u+n c
$$

The functions in the previous result are called fixed points of the Lax operator with critical value $c$. These fixed points are solutions of the following discrete-time Hamilton-Jacobi equation associated to $V$ :

$$
\sup _{x_{1} \in M} \inf _{y_{1} \in N}\left\{u\left(x_{1}, y_{1}\right)-u(x, y)-V\left(x, y, x_{1}, y_{1}\right)\right\}=c .
$$

See [11] for a minimal discrete-time case.

In the third section, we will consider an infinite horizon problem, which will help us to find a different characterization of critical value $c$. To this end, we define the lower Peierls barrier $h_{k}^{-}$as follows, given a real number $k$ and an initial state $(x, y) \in M \times N$ :

$$
h_{k}^{-}(x, y):=\liminf _{n \rightarrow \infty}\left\{\sup _{\Theta(n)} \inf _{\bar{x}(n)} A^{n}(\bar{x}(n), \Theta(n)[\bar{x}(n)])+n k\right\}
$$

where $\bar{x}(n) \in S_{M}(x, n)$ and $\Theta(n) \in \mathscr{E}_{N}(x, y, n)$, see $[3,12,13]$.

It is possible to prove that there exists a number $c^{-} \in \mathbb{R}$, such that

$$
c^{-}=\inf \left\{k \in \mathbb{R}: h_{k}^{-}=+\infty\right\}=\sup \left\{k \in \mathbb{R}: h_{k}^{-}=-\infty\right\},
$$

where the value of $h_{k}^{-}$does not depend on $(x, y) \in M \times N$. The critical value $c$ has a new interpretation characterized by the following result.

Theorem 2. If $M$ and $N$ are compact metric spaces, $V: M \times$ $N \times M \times N \rightarrow \mathbb{R}$ is a Lipschitz function, and $(x, y) \in M \times N$, then $h_{c^{-}}^{-}(x, y) \in \mathbb{R}$ and $c^{-}=c$, where $c$ is the critical value.
Analogously, we can define the upper Peierls barrier $h_{k}^{+}$ 


$$
\begin{aligned}
& h_{k}^{+}(x, y) \\
& \quad:=\limsup _{n \rightarrow \infty}\left\{\sup _{\Theta(n)} \inf _{\bar{x}(n)} A^{n}(\bar{x}(n), \Theta(n)[\bar{x}(n)])+n k\right\},
\end{aligned}
$$

for which we can obtain similar results.

Considering that $h_{c}^{-}(x, y) \in \mathbb{R}$ and $h_{c}^{+}(x, y) \in \mathbb{R}$, for every $(x, y) \in M \times N$, we prove that

$$
\sup _{n \in \mathbb{Z}^{+}}\left|n c+\sup _{\Theta(n)} \inf _{\bar{x}(n)} A^{n}(\bar{x}(n), \Theta(n)[\bar{x}(n)])\right|<+\infty,
$$

and therefore,

$$
-c=\lim _{n \rightarrow \infty} \sup _{\Theta(n)} \inf _{\bar{x}(n)} \frac{1}{n} A^{n}(\bar{x}(n), \Theta(n)[\bar{x}(n)]) .
$$

In addition, using a solution of the Hamilton-Jacobi equation, we can find an optimal strategy, from which optimal sequences are obtained for both players for all times. In particular, we prove the following result.

Theorem 3. If $M$ and $N$ are compact metric spaces, $V: M \times$ $N \times M \times N \rightarrow \mathbb{R}$ is a Lipschitz function, and $(x, y) \in M \times N$, then there are sequences $\left\{x_{i}^{*}\right\}_{i=0}^{\infty} \subset M$ and $\left\{y_{i}^{*}\right\}_{i=0}^{\infty} \subset N$ such that

$$
\sup _{n \in \mathbb{Z}^{+}}\left|n c+A^{n}\left(\bar{x}^{*}(n), \bar{y}^{*}(n)\right)\right|<+\infty,
$$

where $\bar{x}^{*}(n):=\left\{x_{i}^{*}\right\}_{i=0}^{n} \in S_{M}(x, n)$ and $\bar{y}^{*}(n):=\left\{y_{i}^{*}\right\}_{i=0}^{n} \in$ $S_{N}(y, n)$, where $c$ is the critical value.

From this, it follows immediately that

$$
-c=\lim _{n \rightarrow \infty} \frac{1}{n} A^{n}\left(\bar{x}^{*}(n), \bar{y}^{*}(n)\right) .
$$

The sequences obtained in the previous result satisfy the Bellman principle of optimality, because from each state $\left(x_{i}^{*}, y_{i}^{*}\right)$ in the optimal trajectory, the complement remains optimal. The optimality of the remaining decisions from $\left(x_{i}^{*}, y_{i}^{*}\right)$ depends on this last state, which is the result of the previous decisions.

In last section, we will show another characterization for the solutions of the Hamilton-Jacobi equation.

For $k \in \mathbb{R}$, a function $g \in C(M \times N, \mathbb{R})$ is called $k$ supersolution if

$$
\mathscr{L}^{1} g \geq g+k .
$$

Analogously, a function $g \in C(M \times N, \mathbb{R})$ is called $k$ subsolution if

$$
\mathscr{L}^{1} g \leq g+k .
$$

The function $g$ is a $k$-solution if $g$ is $k$-supersolution and $k$ subsolution (see [4]).

Clearly, $g$ is a $k$-solution if and only if $g$ is a fixed point of the Lax operator with critical value $k$. According to Theorem 1, if there exists a unique constant for fixed points of the Lax operator, then there is only one kind of $k$-solutions, in fact for $k=c$. The $c$-solutions are called critical solutions. From this, we get another characterization of $c$, which appears in the following result.
Theorem 4. If $M$ and $N$ are compact metric spaces, $V: M \times$ $N \times M \times N \rightarrow \mathbb{R}$ is a Lipschitz function, and $u \in C(M \times N, \mathbb{R})$ is a fixed point of the Lax operator $\mathscr{L}^{n}$ with critical valuec $\in \mathbb{R}$, then

$$
c=\sup S_{U}(u)=\inf S_{L}(u),
$$

where

$$
\begin{gathered}
S_{U}(u):=\{k \in \mathbb{R}: u \text { is } k \text {-supersolution }\}, \\
S_{L}(u):=\{k \in \mathbb{R}: u \text { is } k \text {-subsolution }\} .
\end{gathered}
$$

Finally, we will show that $-h_{c}^{-}$is a critical solution and therefore a solution of the Hamilton-Jacobi equation.

\section{Lax Operator}

Let $M$ and $N$ be compact metric spaces, $V: M \times N \times M \times$ $N \rightarrow \mathbb{R}$ a Lipschitz function, and $n \in \mathbb{Z}^{+}$. Consider a zerosum game of two players, $J_{M}$ and $J_{N}$, with finite horizon $n$, where $M$ and $N$ represent the state spaces and sequences $\bar{x}(n)=\left\{x_{0}, x_{1}, \ldots, x_{n}\right\} \subset M$ and $\bar{y}(n)=\left\{y_{0}, y_{1}, \ldots, y_{n}\right\} \subset N$ determine the state trajectories, respectively, for players $J_{M}$ and $J_{N}$. The function $A^{n}(\bar{x}(n), \bar{y}(n))$, defined by

$$
A^{n}(\bar{x}(n), \bar{y}(n)):=\sum_{i=0}^{n-1} V\left(x_{i}, y_{i}, x_{i+1}, y_{i+1}\right),
$$

is the game action. For $g \in C(M \times N, \mathbb{R})$ and $(x, y) \in M \times N$, the game payoff $P_{g}: S_{M}(x, n) \times S_{N}(y, n) \rightarrow \mathbb{R}$ is defined by

$$
P_{g}(\bar{x}(n), \bar{y}(n)):=g\left(x_{n}, y_{n}\right)-A^{n}(\bar{x}(n), \bar{y}(n)) .
$$

If $\mathscr{C}_{N}(x, y, n)$ is the set of progressive strategies for player $J_{N}$, the Lax operator is the lower game value, defined by

$$
\mathscr{L}^{n} g(x, y):=\inf _{\Theta(n)} \sup _{\bar{x}(n)} P_{g}(\bar{x}(n), \Theta(n)[\bar{x}(n)]),
$$

with $\bar{x}(n) \in S_{M}(x, n)$ and $\Theta(n) \in \mathscr{E}_{N}(x, y, n)$. We prove the following regularity property for the Lax operator.

Lemma 5. The Lax operator $\mathscr{L}^{n} g$ is K-Lipschitz, for any $n \in$ $\mathbb{Z}^{+}$and $g \in C(M \times N, \mathbb{R})$, where $K$ is the Lipschitz constant of $V$.

Proof. We will consider $(x, y),(z, w) \in M \times N, g \in C(M \times$ $N, \mathbb{R})$, and $\varepsilon>0$. There exists $\Theta^{1}(n) \in \mathscr{E}_{N}(z, w, n)$ such that

$$
\mathscr{L}^{n} g(z, w)>\sup _{\bar{z}(n)} P_{g}\left(\bar{z}(n), \Theta^{1}(n)[\bar{z}(n)]\right)-\varepsilon,
$$

where $\bar{z}(n) \in S_{M}(z, n)$.

For a finite sequence $\bar{x}(n)=\left\{x, x_{1}, \ldots, x_{n}\right\} \in S_{M}(x, n)$, we define $\Theta^{2}(n) \in \mathscr{E}_{N}(x, y, n)$ as

$$
\Theta^{2}(n)[\bar{x}(n)]_{i}= \begin{cases}y, & i=0 \\ \Theta^{1}(n)\left[\left\{z, x_{1}, \ldots, x_{n}\right\}\right]_{i}, & 1 \leq i \leq n .\end{cases}
$$


For this strategy, we have

$$
\mathscr{L}^{n} g(x, y) \leq \sup _{\bar{x}(n)} P_{g}\left(\bar{x}(n), \Theta^{2}(n)[\bar{x}(n)]\right),
$$

where $\bar{x}(n) \in S_{M}(x, n)$. It follows that there is $\bar{x}^{*}(n)=$ $\left\{x, x_{1}^{*}, \ldots, x_{n}^{*}\right\}$ such that

$$
\mathscr{L}^{n} g(x, y)<P_{g}\left(\bar{x}^{*}(n), \Theta^{2}(n)\left[\bar{x}^{*}(n)\right]\right)+\varepsilon
$$

We define $\bar{z}^{*}(n) \in S_{M}(z, n)$ as $\bar{z}^{*}(n)=\left\{z, x_{1}^{*}, \ldots, x_{n}^{*}\right\}$. Combining (28) with (31), we obtain

$$
\begin{aligned}
\mathscr{L}^{n} g(x, y)-\mathscr{L}^{n} g(z, w) \\
<P_{g}\left(\bar{x}^{*}(n), \Theta^{2}(n)\left[\bar{x}^{*}(n)\right]\right) \\
\quad-P_{g}\left(\bar{z}^{*}(n), \Theta^{1}(n)\left[\bar{z}^{*}(n)\right]\right)+2 \varepsilon .
\end{aligned}
$$

Since $x_{i}^{*}=z_{i}^{*}$ and $\Theta^{2}(n)\left[\bar{x}^{*}(n)\right]_{i}=\Theta^{1}(n)\left[\bar{z}^{*}(n)\right]_{i}$, for $1 \leq i \leq$ $n$, we have

$$
\begin{aligned}
\mathscr{L}^{n} g(x, y)-\mathscr{L}^{n} g(z, w) \\
<V\left(z, w, x_{1}^{*}, \Theta^{1}(n)\left[\bar{z}^{*}(n)\right]_{1}\right) \\
\quad-V\left(x, y, x_{1}^{*}, \Theta^{1}(n)\left[\bar{z}^{*}(n)\right]_{1}\right)+2 \varepsilon \\
\leq K d[(x, y),(z, w)]+2 \varepsilon
\end{aligned}
$$

where $K$ is the Lipschitz constant of $V$ and $d$ is a distance in $M \times N$. Similarly, we obtain the opposite inequality. Thus,

$$
\left|\mathscr{L}^{n} g(x, y)-\mathscr{L}^{n} g(z, w)\right| \leq K d[(x, y),(z, w)]
$$

From above result, we prove the semigroup property for the Lax operator, also called the Principle of Dynamic Programming.

Proposition 6 (Semigroup Property). For $m, n \in \mathbb{Z}^{+}$,

$$
\begin{aligned}
& \mathscr{L}^{m+n} g(x, y)= \mathscr{L}^{m} \circ \mathscr{L}^{n} g(x, y) \\
&=\inf _{\Theta(m)} \sup _{\bar{x}(m)}\left\{\mathscr{L}^{n} g\left(x_{m}, \Theta(m)[\bar{x}(m)]_{m}\right)\right. \\
& \\
&\left.\quad-A^{m}(\bar{x}(m), \Theta(m)[\bar{x}(m)])\right\},
\end{aligned}
$$

where $\Theta(m) \in \mathscr{E}_{N}(x, y, m)$ and $\bar{x}(m) \in S_{M}(x, m)$.

Proof. We will consider $\varepsilon>0$ and $g \in C(M \times N, \mathbb{R})$. To prove the relation $\mathscr{L}^{m+n} g=\mathscr{L}^{m} \circ \mathscr{L}^{n} g$, we will see that, for every $(x, y) \in M \times N$, the inequalities $\mathscr{L}^{m+n} g(x, y) \leq \mathscr{L}^{m}$ 。 $\mathscr{L}^{n} g(x, y)$ and $\mathscr{L}^{m+n} g(x, y) \geq \mathscr{L}^{m} \circ \mathscr{L}^{n} g(x, y)$ take place simultaneously.
(I) Let us first prove that $\mathscr{L}^{m+n} g \leq \mathscr{L}^{m} \circ \mathscr{L}^{n} g$. To do this, take $\Theta^{1}(m) \in \mathscr{E}_{N}(x, y, m)$ such that

$$
\begin{aligned}
\mathscr{L}^{m}\left(\mathscr{L}^{n} g\right)(x, y) & \\
>\sup _{\bar{x}(m)} & \left\{\mathscr{L}^{n} g\left(x_{m}, \Theta^{1}(m)[\bar{x}(m)]_{m}\right)\right. \\
& \left.-A^{m}\left(\bar{x}(m), \Theta^{1}(m)[\bar{x}(m)]\right)\right\}-\varepsilon,
\end{aligned}
$$

where $\bar{x}(m) \in S_{M}(x, m)$.

On the other hand, for each $(z, w) \in M \times N$,

$$
\begin{aligned}
\mathscr{L}^{n} g(z, w)=\inf _{\Theta(n)} \sup _{\bar{z}(n)}\{ & g\left(z_{n}, \Theta(n)[\bar{z}(n)]_{n}\right) \\
& \left.-A^{n}(\bar{z}(n), \Theta(n)[\bar{z}(n)])\right\},
\end{aligned}
$$

where $\Theta(n) \in \mathscr{E}_{N}(z, w, n)$ and $\bar{z}(n) \in S_{M}(z, n)$. Hence, there is $\Theta_{(z, w)}^{2}(n) \in \mathscr{E}_{N}(z, w, n)$ such that

$$
\begin{aligned}
\mathscr{L}^{n} g(z, w)>\sup _{\bar{z}(n)}\{ & g\left(z_{n}, \Theta_{(z, w)}^{2}(n)[\bar{z}(n)]_{n}\right) \\
& \left.-A^{n}\left(\bar{z}(n), \Theta_{(z, w)}^{2}(n)[\bar{z}(n)]\right)\right\}-\varepsilon .
\end{aligned}
$$

We will define a $\Theta^{*}(m+n) \in \mathscr{E}_{N}(x, y, m+n)$ using the previous strategy $\Theta^{1}(m)$ and the family $\Theta_{(z, w)}^{2}(n)$. Given a sequence in $S_{M}(x, m+n)$, the associated sequence in $S_{N}(y, m+$ $n)$ is defined as follows: the fist $m$ terms are given by the $\Theta^{1}(m)$ strategy and the second part is given by $\Theta_{\left(z^{*}, w^{*}\right)}^{2}(n)$, for a $\operatorname{specific}\left(z^{*}, w^{*}\right)$. More precisely, for $\bar{x}(m+n) \in S_{M}(x, m+n)$,

$$
\begin{aligned}
& \Theta^{*}(m+n)[\bar{x}(m+n)]_{i} \\
& = \begin{cases}\Theta^{1}(m)\left[\bar{x}^{1}(m)\right]_{i}, & 0 \leq i \leq m \\
\Theta_{\left(z^{*}, w^{*}\right)}^{2}(n)\left[\bar{x}^{2}(n)\right]_{i-m}, & m+1 \leq i \leq m+n,\end{cases}
\end{aligned}
$$

where $\bar{x}^{1}(m)=\left\{x, x_{1}, \ldots, x_{m}\right\},\left(z^{*}, w^{*}\right)=\left(x_{m}\right.$, $\left.\Theta^{1}(m)\left[\bar{x}^{1}(m)\right]_{m}\right), \quad \bar{x}^{2}(n)=\left\{z^{*}, x_{m+1}, \ldots, x_{m+n}\right\}, \quad$ and $\Theta_{\left(z^{*}, w^{*}\right)}^{2}(n) \in \mathscr{E}_{N}\left(z^{*}, w^{*}, n\right)$. Taking any $\bar{x}(m+n) \in S_{M}(x, m+$ $n)$ and combining (36) with (38), we obtain

$$
\begin{aligned}
& \mathscr{L}^{m}\left(\mathscr{L}^{n} g\right)(x, y) \\
&> \mathscr{L}^{n} g\left(x_{m}, \Theta^{1}(m)\left[\bar{x}^{1}(m)\right]_{m}\right) \\
&-A^{m}\left(\bar{x}^{1}(m), \Theta^{1}(m)\left[\bar{x}^{1}(m)\right]\right)-\varepsilon \\
&> g\left(x_{m+n}, \Theta_{\left(z^{*}, w^{*}\right)}^{2}(n)\left[\bar{x}^{2}(n)\right]_{n}\right) \\
&-A^{n}\left(\bar{x}^{2}(n), \Theta_{\left(z^{*}, w^{*}\right)}^{2}(n)\left[\bar{x}^{2}(n)\right]\right) \\
&-A^{m}\left(\bar{x}^{1}(m), \Theta^{1}(m)\left[\bar{x}^{1}(m)\right]\right)-2 \varepsilon \\
&= g\left(x_{m+n}, \Theta^{*}(m+n)[\bar{x}(m+n)]_{m+n}\right) \\
&-A^{m+n}\left(\bar{x}(m+n), \Theta^{*}(m+n)[\bar{x}(m+n)]\right)-2 \varepsilon .
\end{aligned}
$$


Therefore,

$$
\begin{aligned}
\sup _{\bar{x}(m+n)} & \left\{g\left(x_{m+n}, \Theta^{*}(m+n)[\bar{x}(m+n)]_{m+n}\right)\right. \\
& \left.-A^{m+n}\left(\bar{x}(m+n), \Theta^{*}(m+n)[\bar{x}(m+n)]\right)\right\} \\
\leq & \mathscr{L}^{m}\left(\mathscr{L}^{n} g\right)(x, y)+2 \varepsilon,
\end{aligned}
$$

with $\bar{x}(m+n) \in S_{M}(x, m+n)$. This implies that

$$
\begin{aligned}
& \mathscr{L}^{m+n} g(x, y) \\
& =\inf _{\Theta(m+n)} \sup _{\bar{x}(m+n)}\left\{g\left(x_{m+n}, \Theta(m+n)[\bar{x}(m+n)]_{m+n}\right)\right. \\
& \left.\quad-A^{m+n}(\bar{x}(m+n), \Theta(m+n)[\bar{x}(m+n)])\right\} \\
& \leq \mathscr{L}^{m}\left(\mathscr{L}^{n} g\right)(x, y)+2 \varepsilon,
\end{aligned}
$$

and consequently $\mathscr{L}^{m+n} g \leq \mathscr{L}^{m} \circ \mathscr{L}^{n} g$.

(II) It remains to prove that $\mathscr{L}^{m+n} g \geq \mathscr{L}^{m} \circ \mathscr{L}^{n} g$. To this end, we will consider $\Theta^{*}(m+n) \in \mathscr{E}_{N}(x, y, m+n)$ such that

$$
\begin{aligned}
\mathscr{L}^{m+n} g(x, y) & \\
>\sup _{\bar{x}(m+n)} & \left\{g\left(x_{m+n}, \Theta^{*}(m+n)[\bar{x}(m+n)]_{m+n}\right)\right. \\
& \left.\quad-A^{m+n}\left(\bar{x}(m+n), \Theta^{*}(m+n)[\bar{x}(m+n)]\right)\right\}-\varepsilon,
\end{aligned}
$$

where $\bar{x}(m+n) \in S_{M}(x, m+n)$.

We will define $\Theta^{1}(m) \in \mathscr{E}_{N}(x, y, m)$ as the $m$-time restriction of $\Theta^{*}(m+n)$. Given a sequence $\bar{z}(m) \in S_{M}(x, m)$, the associated sequence in $S_{N}(y, m)$ is defined as follows: the $m$ terms are given by the fist $m$ terms of $\Theta^{*}(m+n)$ strategy applied to some extension of $\bar{z}(m)$ in $S_{M}(x, m+n)$, adding any sequence $\bar{w}(n) \in S_{M}\left(z_{m}, n\right)$. Since $\Theta^{*}(m+n)$ is a strategy, it follows that the function $\Theta^{1}(m)$ is well defined and it is a strategy. More precisely, for $\bar{z}(m) \in S_{M}(x, m)$, taking any $\bar{w}(n)=\left\{z_{m}, w_{1}, \ldots, w_{n}\right\} \in S_{M}\left(z_{m}, n\right)$, we define $\Theta^{1}(m) \in$ $\mathscr{E}_{N}(x, y, m)$ as

$$
\Theta^{1}(m)[\bar{z}(m)]_{i}=\Theta^{*}(m+n)[\bar{x}(m+n)]_{i},
$$

for $i=0,1, \ldots, m$, where $\bar{x}(m+n)=\left\{x, z_{1}, \ldots, z_{m}, w_{1}, \ldots\right.$, $\left.w_{n}\right\}$. So

$$
\begin{aligned}
& \mathscr{L}^{m}\left(\mathscr{L}^{n} g\right)(x, y) \\
& \leq \sup _{\bar{z}(m)}\left\{\mathscr{L}^{n} g\left(z_{m}, \Theta^{1}(m)[\bar{z}(m)]_{m}\right)\right. \\
&\left.\quad-A^{m}\left(\bar{z}(m), \Theta^{1}(m)[\bar{z}(m)]\right)\right\},
\end{aligned}
$$

where $\bar{z}(m) \in S_{M}(x, m)$. Consequently, there is $\bar{z}^{1}(m) \in$ $S_{M}(x, m)$ such that

$$
\begin{aligned}
\mathscr{L}^{m}\left(\mathscr{L}^{n} g\right)(x, y)< & \mathscr{L}^{n} g\left(z_{m}^{1}, \Theta^{1}(m)\left[\bar{z}^{1}(m)\right]_{m}\right) \\
& -A^{m}\left(\bar{z}^{1}(m), \Theta^{1}(m)\left[\bar{z}^{1}(m)\right]\right)+\varepsilon .
\end{aligned}
$$

Once that $z_{m}^{1} \in M$ is fixed so will be $\Theta^{1}(m)\left[\bar{z}^{1}(m)\right]_{m} \in N$. We now define $\Theta^{2}(n) \in \mathscr{E}_{N}\left(z_{m}^{1}, \Theta^{1}(m)\left[\bar{z}^{1}(m)\right]_{m}, n\right)$, for $\bar{w}(n) \in$ $S_{M}\left(z_{m}^{1}, n\right)$, as

$$
\Theta^{2}(n)[\bar{w}(n)]_{i}=\Theta^{*}(m+n)[\bar{x}(m+n)]_{i+m},
$$

for $i=0,1, \ldots, n$, where $\bar{x}(m+n)=\left\{x, z_{1}^{1}, \ldots, z_{m}^{1}, w_{1}, \ldots\right.$, $\left.w_{n}\right\}$. We have

$$
\begin{aligned}
\mathscr{L}^{n} g\left(z_{m}^{1}, \Theta^{1}(m)\left[\bar{z}^{1}(m)\right]_{m}\right) \\
\leq \sup _{\bar{w}(n)}\left\{g\left(w_{n}, \Theta^{2}(n)[\bar{w}(n)]_{n}\right)\right. \\
\left.\quad-A^{n}\left(\bar{w}(n), \Theta^{2}(n)[\bar{w}(n)]\right)\right\},
\end{aligned}
$$

where $\bar{w}(n) \in S_{M}\left(z_{m}^{1}, n\right)$. Therefore, there is $\bar{z}^{2}(n) \in$ $S_{M}\left(z_{m}^{1}, n\right)$ such that

$$
\begin{aligned}
\mathscr{L}^{n} g\left(z_{m}^{1}, \Theta^{1}(m)\left[\bar{z}^{1}(m)\right]_{m}\right) \\
<g\left(z_{n}^{2}, \Theta^{2}(n)\left[\bar{z}^{2}(n)\right]_{n}\right) \\
\quad-A^{n}\left(\bar{z}^{2}(n), \Theta^{2}(n)\left[\bar{z}^{2}(n)\right]\right)+\varepsilon .
\end{aligned}
$$

Define $\bar{x}^{*}(m+n) \in S_{M}(x, m+n)$ as

$$
\bar{x}^{*}(m+n)=\left\{x, z_{1}^{1}, \ldots, z_{m}^{1}, z_{1}^{2}, \ldots, z_{n}^{2}\right\} .
$$

Combining (43) and (46) with (49) we obtain:

$$
\begin{aligned}
& \mathscr{L}^{m}\left(\mathscr{L}^{n} g\right)(x, y) \\
&< \mathscr{L}^{n} g\left(z_{m}^{1}, \Theta^{1}(m)\left[\bar{z}^{1}(m)\right]_{m}\right) \\
&-A^{m}\left(\bar{z}^{1}(m), \Theta^{1}(m)\left[\bar{z}^{1}(m)\right]\right)+\varepsilon \\
&< g\left(z_{n}^{2}, \Theta^{2}(n)\left[\bar{z}^{2}(n)\right]_{n}\right)-A^{n}\left(\bar{z}^{2}(n), \Theta^{2}(n)\left[\bar{z}^{2}(n)\right]\right) \\
&-A^{m}\left(\bar{z}^{1}(m), \Theta^{1}(m)\left[\bar{z}^{1}(m)\right]\right)+2 \varepsilon \\
&= g\left(x_{m+n}^{*}, \Theta^{*}(m+n)\left[\bar{x}^{*}(m+n)\right]_{m+n}\right) \\
&-A^{m+n}\left(\bar{x}^{*}(m+n), \Theta^{*}(m+n)\left[\bar{x}^{*}(m+n)\right]\right)+2 \varepsilon \\
&< \mathscr{L}^{m+n} g(x, y)+3 \varepsilon,
\end{aligned}
$$

for this reason $\mathscr{L}^{m} \circ \mathscr{L}^{n} \leq \mathscr{L}^{m+n}$.

Lemma 7. If $g, f \in C(M \times N, \mathbb{R})$, then

(1) (monotony) if $g \leq f$, then $\mathscr{L}^{n} g \leq \mathscr{L}^{n} f$;

(2) if $k \in \mathbb{R}$, then $\mathscr{L}^{n}(k+g)=k+\mathscr{L}^{n} g$;

(3) (weak contraction) $\left\|\mathscr{L}^{n} g-\mathscr{L}^{n} f\right\|_{\infty} \leq\|g-f\|_{\infty}$.

Proof. The first two properties are a direct consequence of the definition. For last the claim, choose $(x, y) \in M \times N$, then

$$
f(x, y)+\|g-f\|_{\infty} \leq g(x, y) \leq f(x, y)+\|g-f\|_{\infty} .
$$


The previous items imply that

$$
\begin{aligned}
\mathscr{L}^{n} f(x, y)+\|g-f\|_{\infty} & \leq \mathscr{L}^{n} g(x, y) \\
& \leq \mathscr{L}^{n} f(x, y)+\|g-f\|_{\infty} .
\end{aligned}
$$

So $\left|\left(\mathscr{L}^{n} g-\mathscr{L}^{n} f\right)(x, y)\right| \leq\|g-f\|_{\infty}$, for every $(x, y) \in M \times N$. As a result,

$$
\left\|\mathscr{L}^{n} g-\mathscr{L}^{n} f\right\|_{\infty} \leq\|g-f\|_{\infty} .
$$

Theorem 1 is analogue to Weak KAM Theorem. We use the same argument of Fathi (see [3]) to prove the existence of fixed points of this discrete-time case of the Lax operator.

According to this result, we say that $u$ is a fixed point of the Lax operator with critical value $c$.

Proof. (I) To prove the existence of a fixed point, we will use the same argument used by Fathi ([3]) for the continuoustime case.

Let $E$ be the quotient of the set of continuous functions $C(M \times N, \mathbb{R})$ modulo constants, $E=C(M \times N, \mathbb{R}) / \mathbb{R} \cdot 1$. The norm $\|\cdot\|: E \rightarrow \mathbb{R}$ is defined as

$$
\|[u]\|=\inf _{a \in \mathbb{R}}\|u+a\|_{\infty}
$$

for $[u] \in E$. Then, $(E,\|\cdot\|)$ is a Banach space. We define the operator $\widehat{\mathscr{L}}^{n}: E \rightarrow E$ as

$$
\widehat{\mathscr{L}}^{n}[u]=\left[\mathscr{L}^{n} u\right],
$$

which satisfies the weak contraction property. Thus, $[u] \mapsto$ $\lambda \widehat{\mathscr{L}}^{n}[u]$, with $\lambda \in(0,1)$, is a contraction; so there are fixed points $[u]_{\lambda} \in E$ of $\lambda \widehat{\mathscr{L}}^{n}$. As $\mathscr{L}^{n}$ is equi-Lipschitz, since Arzela-Ascoli theorem, it follows that $\left\{[u]_{\lambda}\right\}$ has a uniformly convergent subsequence when $\lambda \rightarrow 1$. Then, $\widehat{\mathscr{L}}^{n}$ has a fixed point $[u]_{*} \in E$, which in terms of $\mathscr{L}^{n}$ means that there is a constant $c_{n} \in \mathbb{R}$ such that

$$
\mathscr{L}^{n} u_{*}=u_{*}+c_{n}
$$

for each positive integer $n$. From the semigroup property, there is $c \in \mathbb{R}$ such that

$$
\mathscr{L}^{n} u_{*}=u_{*}+n c
$$

for all positive integers $n$.

Function $u_{*}$ is a Lipschitz function, because $\mathscr{L}^{n} u_{*}$ is also a Lipschitz function. In fact, according to Lemma $5, u_{*}$ is $K$ Lipschitz, where $K$ is the Lipschitz constant of $V$.

(II) It remains to prove that the number $c$ is unique. Let $\varepsilon$ be positive. Suppose there are $u_{i} \in C(M \times N, \mathbb{R})$ and $c_{i} \in \mathbb{R}$, $i=1,2$, such that $\mathscr{L}^{n} u_{i}=u_{i}+n c_{i}$, with $c_{1}>c_{2}$. Choose $\Theta^{*}(n) \in \mathscr{E}_{N}(x, y, n)$ such that

$$
\begin{aligned}
\mathscr{L}^{n} u_{2}(x, y)>\sup _{\bar{x}(n)}\left\{u_{2}\left(x_{n}, \Theta^{*}(n)[\bar{x}(n)]_{n}\right)\right. \\
\left.-A^{n}\left(\bar{x}(n), \Theta^{*}(n)[\bar{x}(n)]\right)\right\}-\varepsilon .
\end{aligned}
$$

For this strategy, we also have that

$$
\begin{aligned}
\mathscr{L}^{n} u_{1}(x, y) \leq \sup _{\bar{x}(n)}\left\{u_{1}\left(x_{n}, \Theta^{*}(n)[\bar{x}(n)]_{n}\right)\right. \\
\left.-A^{n}\left(\bar{x}(n), \Theta^{*}(n)[\bar{x}(n)]\right)\right\} .
\end{aligned}
$$

So there is $\bar{x}^{*}(n) \in S_{M}(x, n)$, such that

$$
\begin{aligned}
\mathscr{L}^{n} u_{1}(x, y)< & u_{1}\left(x_{n}^{*}, \Theta^{*}(n)\left[\bar{x}^{*}(n)\right]_{n}\right) \\
& -A^{n}\left(\bar{x}^{*}(n), \Theta^{*}(n)\left[\bar{x}^{*}(n)\right]\right)+\varepsilon .
\end{aligned}
$$

Therefore, using the hypothesis, we obtain

$$
\begin{aligned}
u_{1}(x, y)+n c_{1}< & u_{1}\left(x_{n}^{*}, \Theta^{*}(n)\left[\bar{x}^{*}(n)\right]_{n}\right) \\
& -A^{n}\left(\bar{x}^{*}(n), \Theta^{*}(n)\left[\bar{x}^{*}(n)\right]\right)+\varepsilon, \\
u_{2}(x, y)+n c_{2}> & u_{2}\left(x_{n}^{*}, \Theta^{*}(n)\left[\bar{x}^{*}(n)\right]_{n}\right) \\
& -A^{n}\left(\bar{x}^{*}(n) \Theta^{*}(n)\left[\bar{x}^{*}(n)\right]\right)-\varepsilon .
\end{aligned}
$$

Considering the subtraction of the previous inequalities, we obtain following relations:

$$
\begin{aligned}
\inf _{M \times N} & \left(u_{1}-u_{2}\right)+n\left(c_{1}-c_{2}\right) \\
\leq & u_{1}(x, y)+n c_{1}-u_{2}(x, y)-n c_{2} \\
< & u_{1}\left(x_{n}^{*}, \Theta^{*}(m+n)\left[\bar{x}^{*}(n)\right]_{n}\right) \\
& -u_{2}\left(x_{n}^{*}, \Theta^{*}(m+n)\left[\bar{x}^{*}(n)\right]_{n}\right)+2 \varepsilon \\
\leq & \sup _{M \times N}\left(u_{1}-u_{2}\right)+2 \varepsilon,
\end{aligned}
$$

then

$$
n\left(c_{1}-c_{2}\right)<\sup _{M \times N}\left(u_{1}-u_{2}\right)-\inf _{M \times N}\left(u_{1}-u_{2}\right)+2 \varepsilon .
$$

On the other hand, because $c_{1}>c_{2}$, we have $n\left(c_{1}-c_{2}\right) \rightarrow \infty$ when $n \rightarrow \infty$, which is a contradiction.

Considering the one-step case (see [8]), we can rewrite the Lax operator as

$$
\mathscr{L}^{1} g(x, y)=\sup _{x_{1} \in M} \inf _{y_{1} \in N}\left\{g\left(x_{1}, y_{1}\right)-V\left(x, y, x_{1}, y_{1}\right)\right\} .
$$

If $u \in C(M \times N, \mathbb{R})$ is a fixed point with critical value $c$, then $u$ is a solution for the following discrete-time analog of the Hamilton-Jacobi equation:

$$
\sup _{x_{1} \in M} \inf _{y_{1} \in N}\left\{u\left(x_{1}, y_{1}\right)-u(x, y)-V\left(x, y, x_{1}, y_{1}\right)\right\}=c \text {. }
$$

Conversely, if $u$ is a solution of the previous HamiltonJacobi equation, then $u$ is a fixed point of the Lax operator $\mathscr{L}^{1}$ with critical value $c$. According to semigroup property and relation 2 in Lemma 7, it follows that $u$ is a fixed point of $\mathscr{L}^{n}$ with critical value $c$, for all $n \in \mathbb{Z}^{+}$. 


\section{Peierls Barrier}

Let us now study an infinite horizon problem for players $J_{M}$ and $J_{N}$. Let $M$ and $N$ be compact metric spaces, and let $V$ : $M \times N \times M \times N \rightarrow \mathbb{R}$ be a Lipschitz function. For $n \in \mathbb{Z}^{+}$, let us consider the function $h(n)$ defined as

$$
h(n)(x, y):=\sup _{\Theta(n)} \inf _{\bar{x}(n)} A^{n}(\bar{x}(n), \Theta(n)[\bar{x}(n)]),
$$

where $\bar{x}(n) \in S_{M}(x, n)$ and $\Theta(n) \in \mathscr{E}_{N}(x, y, n)$.

For $k \in \mathbb{R}$, the lower Peierls barrier $h_{k}^{-}: M \times N \rightarrow \mathbb{R}$ is

$$
h_{k}^{-}(x, y):=\liminf _{n \rightarrow \infty}\{h(n)(x, y)+k n\} .
$$

Proposition 8. For every $k \in \mathbb{R}$, one has the following:

(1) if $h_{k}^{-}(x, y)=-\infty$ for some $(x, y) \in M \times N$, then $h_{k}^{-}(z, w)=-\infty$, for all $(z, w) \in M \times N$;

(2) if $h_{k}^{-}(x, y)=+\infty$ for some $(x, y) \in M \times N$, then $h_{k}^{-}(z, w)=+\infty$, for all $(z, w) \in M \times N$.

Proof. Let $B$ be positive and consider $C>\sup V-\inf V+B$.

(1) Let us take $(z, w) \in M \times N$ and suppose that $h_{k}^{-}(x, y)=$ $-\infty$. Hence, there is $n_{j} \uparrow \infty$ such that

$$
\lim _{j \rightarrow \infty}\left\{h\left(n_{j}\right)(x, y)+k n_{j}\right\}=-\infty .
$$

Therefore, there is $J \in \mathbb{Z}^{+}$such that if $j \geq J$, then

$$
h\left(n_{j}\right)(x, y)+k n_{j}<-C .
$$

Let $\Theta\left(n_{j}\right) \in \mathscr{E}_{N}\left(z, w, n_{j}\right)$ be a strategy. We define $\Theta^{0}\left(n_{j}\right) \in \mathscr{E}_{N}\left(x, y, n_{j}\right)$ as follows: for $\bar{x}\left(n_{j}\right)=\{x$, $\left.x_{1}, \ldots, x_{n_{j}}\right\} \in S_{M}\left(x, n_{j}\right)$,

$$
\begin{aligned}
& \Theta^{0}\left(n_{j}\right)\left[\bar{x}\left(n_{j}\right)\right]_{i} \\
& \quad= \begin{cases}y, & i=0, \\
\Theta\left(n_{j}\right)\left[\left\{z, x_{1}, \ldots, x_{n_{j}}\right\}\right]_{i}, & 1 \leq i \leq n_{j} .\end{cases}
\end{aligned}
$$

Therefore, $\inf _{\bar{x}\left(n_{j}\right)} A^{n_{j}}\left(\bar{x}\left(n_{j}\right), \Theta^{0}\left(n_{j}\right)\left[\bar{x}\left(n_{j}\right)\right]\right)+k n_{j}<$ $-C$. Hence, there is $\bar{x}^{0}\left(n_{j}\right)=\left\{x, x_{1}^{0}, \ldots, x_{n_{j}}^{0}\right\}$ such that

$$
A^{n_{j}}\left(\bar{x}^{0}\left(n_{j}\right), \Theta^{0}\left(n_{j}\right)\left[\bar{x}^{0}\left(n_{j}\right)\right]\right)+k n_{j}<-C .
$$

If we define $\bar{z}^{0}\left(n_{j}\right)=\left\{z, x_{1}^{0}, \ldots, x_{n_{j}}^{0}\right\} \in S_{M}\left(z, n_{j}\right)$, then

$$
\begin{aligned}
A^{n_{j}}\left(\bar{z}^{0}\left(n_{j}\right), \Theta\left(n_{j}\right)\left[\bar{z}^{0}\left(n_{j}\right)\right]\right)+k n_{j} \\
=V\left(z, w, x_{1}^{0}, \Theta\left(n_{j}\right)\left[\bar{z}^{0}\left(n_{j}\right)\right]_{1}\right) \\
\quad-V\left(x, y, x_{1}^{0}, \Theta\left(n_{j}\right)\left[\bar{z}^{0}\left(n_{j}\right)\right]_{1}\right) \\
\quad+A^{n_{j}}\left(\bar{x}^{0}\left(n_{j}\right), \Theta^{0}\left(n_{j}\right)\left[\bar{x}^{0}\left(n_{j}\right)\right]\right)+k n_{j} \\
<\sup V-\inf V-C \\
<-B,
\end{aligned}
$$

in which it follows that $\inf _{\bar{z}\left(n_{j}\right)} A^{n_{j}}\left(\bar{z}\left(n_{j}\right)\right.$, $\left.\Theta\left(n_{j}\right)\left[\bar{z}\left(n_{j}\right)\right]\right)+k n_{j}<-B$, for every $\Theta\left(n_{j}\right) \in \mathscr{E}_{N}(z, w$, $n_{j}$ ), where $j \geq J$. Then,

$$
\lim _{j \rightarrow \infty}\left\{h\left(n_{j}\right)(z, w)+k n_{j}\right\}=-\infty ;
$$

$$
\text { so } h_{k}^{-}(z, w)=-\infty \text {. }
$$

(2) Let us take $(z, w) \in M \times N$ and suppose that $h_{k}^{-}(x, y)=$ $\infty$. There is $N \in \mathbb{Z}^{+}$such that if $n \geq N$, then

$$
h(n)(x, y)+k n>C .
$$

Therefore, there is $\Theta^{0}(n) \in \mathscr{E}_{N}(x, y, n)$ such that

$$
\inf _{\bar{x}(n)} A^{n}\left(\bar{x}(n), \Theta^{0}(n)[\bar{x}(n)]\right)+k n>C .
$$

Taking $\bar{z}(n)=\left\{z, z_{1}, \ldots, z_{n}\right\} \in S_{M}(z, n)$, define $\Theta^{1}(n) \in \mathscr{E}_{N}(z, w, n)$ as

$$
\Theta^{1}(n)[\bar{z}(n)]_{i}= \begin{cases}w, & i=0, \\ \Theta^{0}(n)\left[\left\{x, z_{1}, \ldots, z_{n}\right\}\right]_{i}, & 1 \leq i \leq n .\end{cases}
$$

So, considering $\bar{x}(n)=\left\{x, z_{1}, \ldots, z_{n}\right\} \in S_{M}(x, n)$,

$$
\begin{aligned}
& A^{n}\left(\bar{z}(n), \Theta^{1}(n)[\bar{z}(n)]\right)+k n \\
&=V\left(z, w, z_{1}, \Theta^{1}(n)[\bar{z}(n)]_{1}\right) \\
& \quad-V\left(x, y, z_{1}, \Theta^{0}(n)[\bar{x}(n)]_{1}\right) \\
& \quad+A^{n}\left(\bar{x}(n), \Theta^{0}(n)[\bar{x}(n)]\right)+k n \\
&> \inf V-\sup V+C \\
&> B .
\end{aligned}
$$

Since the previous relation is valid for every $\bar{z}(n) \epsilon$ $S_{M}(z, n)$, it follows that

$$
\inf _{\bar{x}(n)} A^{n}\left(\bar{z}(n), \Theta^{1}(n)[\bar{z}(n)]\right)+k n>B .
$$

Hence,

$$
h_{k}^{-}(z, w)=\lim _{n \rightarrow \infty}\{h(n)(z, w)+k n\}=\infty .
$$

In order to see that $h_{k}^{-}(x, y)$ has a radical change on its values, for a particular value of $k$, we prove the following statement.

Lemma 9. There exists $k>0$ large enough, such that
(1) $h_{-k}^{-}(x, y)=-\infty$.
(2) $h_{k}^{-}(x, y)=+\infty$. 
Proof. Considering $k>\sup V+1$, we have $V-k \leq \sup V-$ $k<-1$. If $\bar{x}(n) \in S_{M}(x, n)$ and $\Theta(n) \in \mathscr{E}_{N}(x, y, n)$, then $A^{n}(\bar{x}(n), \Theta(n)[\bar{x}(n)])-k n<-n$, for each $n \in \mathbb{Z}^{+}$. Hence,

$$
h_{-k}^{-}(x, y)=-\infty
$$

The other relation can be obtained similarly.

It is clear that $h_{k}^{-}$is monotone in $k$.

Lemma 10. Let $k, k_{1}$, and $k_{2}$ be real numbers such that $k_{1}<$ $k<k_{2}$, then

(1) if $h_{k}^{-}(x, y)=-\infty$, then $h_{k_{1}}^{-}(x, y)=-\infty$;

(2) if $h_{k}^{-}(x, y)=+\infty$, then $h_{k_{2}}^{-}(x, y)=+\infty$;

(3) moreover, if $h_{k}^{-}(x, y) \in \mathbb{R}$, then

$$
\begin{aligned}
& h_{k_{1}}^{-}(x, y)=-\infty, \\
& h_{k_{2}}^{-}(x, y)=+\infty .
\end{aligned}
$$

Corollary 11. There exists $c^{-} \in \mathbb{R}$, such that

$$
\inf \left\{k \in \mathbb{R}: h_{k}^{-}=+\infty\right\}=\sup \left\{k \in \mathbb{R}: h_{k}^{-}=-\infty\right\}=c^{-} \text {. }
$$

Proof. From the previous results, it follows that there are

$$
\inf \left\{k \in \mathbb{R}: h_{k}^{-}=+\infty\right\}, \quad \sup \left\{k \in \mathbb{R}: h_{k}^{-}=-\infty\right\} \in \mathbb{R} \text {. }
$$

Given $(x, y) \in M \times N$, choose $k_{1}, k_{2} \in \mathbb{R}$ such that $h_{k_{1}}^{-}(x, y)=$ $-\infty$ and $h_{k_{2}}^{-}(x, y)=+\infty$, then $k_{1} \leq k_{2}$. Therefore, $\sup \{k \in \mathbb{R}$ : $\left.h_{k}^{-}=-\infty\right\} \leq k_{2}$, so

$$
\sup \left\{k \in \mathbb{R}: h_{k}^{-}=-\infty\right\} \leq \inf \left\{k \in \mathbb{R}: h_{k}^{-}=+\infty\right\}
$$

Suppose that $\sup \left\{k \in \mathbb{R}: h_{k}^{-}=-\infty\right\}<\inf \left\{k \in \mathbb{R}: h_{k}^{-}=\right.$ $+\infty\}$, then there are $k_{0} \in \mathbb{R}$ and $\varepsilon>0$ such that $h_{k_{0}}^{-}(x, y) \in \mathbb{R}$ and

$$
\begin{aligned}
\sup \{ & \left.k \in \mathbb{R}: h_{k}^{-}=-\infty\right\} \\
& <k_{0}-\varepsilon<k_{0}+\varepsilon \\
& <\inf \left\{k \in \mathbb{R}: h_{k}^{-}=+\infty\right\} .
\end{aligned}
$$

Lemma 10 shows that $h_{k_{0}-\varepsilon}^{-}=-\infty$ and $h_{k_{0}+\varepsilon}^{-}=+\infty$; this is a contradiction. Hence,

$$
\sup \left\{k \in \mathbb{R}: h_{k}^{-}=-\infty\right\}=\inf \left\{k \in \mathbb{R}: h_{k}^{-}=+\infty\right\}
$$

Theorem 2 provides us a different characterization for the critical value $c$.
Proof. Let $u$ be a fixed point of the Lax operator with critical value $c$. For $(x, y) \in M \times N$ and $n \in \mathbb{Z}^{+}$,

$$
\begin{aligned}
u(x, y)+n c & =\mathscr{L}^{n} u(x, y) \\
= & \inf _{\Theta(n)} \sup _{\bar{x}(n)}\left\{u\left(x_{n}, \Theta(n)[\bar{x}(n)]_{n}\right)\right. \\
& \left.\quad-A^{n}(\bar{x}(n), \Theta(n)[\bar{x}(n)])\right\},
\end{aligned}
$$

where $\bar{x}(n) \in S_{M}(x, n)$ and $\Theta(n) \in \mathscr{E}_{N}(x, y, n)$. Therefore,

$$
\inf u+n c \leq \sup u-\operatorname{supinf}_{\Theta(n)} A^{n}(\bar{x})(\bar{x}(n), \Theta(n)[\bar{x}(n)]) ;
$$

and then, for every $n \in \mathbb{Z}^{+}$,

$$
h(n)(x, y)+c n \leq \sup u-\inf u .
$$

So $h_{c}^{-}(x, y)<+\infty$; on that account, $c \leq c^{-}$. Analogously, from (88), we obtain

$$
\sup u+n c \geq \inf u-\sup _{\Theta(n)} \inf _{\bar{x}(n)} A^{n}(\bar{x}(n), \Theta(n)[\bar{x}(n)]) ;
$$

consequently, for each $n \in \mathbb{Z}^{+}$,

$$
h(n)(x, y)+c n \geq \inf u-\sup u .
$$

Hence, $h_{c}^{-}(x, y)>-\infty$ and in addition $c \geq c^{-}$.

We can introduce the upper Peierls barrier $h_{k}^{+}: M \times N \rightarrow$ $\mathbb{R}$ defined by

$$
h_{k}^{+}(x, y):=\limsup _{n \rightarrow \infty} h(n)(x, y)+k n .
$$

By similar arguments to the ones we made for $h_{k}^{-}$, we obtain analogous properties for $h_{k}^{+}$and the following result.

Theorem 12. If $M$ and $N$ are compact metric spaces, $V: M \times$ $N \times M \times N \rightarrow \mathbb{R}$ is a Lipschitz function, and $(x, y) \in M \times N$, then

$$
c=\inf \left\{k \in \mathbb{R}: h_{k}^{+}=+\infty\right\}=\sup \left\{k \in \mathbb{R}: h_{k}^{+}=-\infty\right\}
$$

and $h_{c}^{+}(x, y) \in \mathbb{R}$, where $c$ is the critical value.

On account of lower and upper Peierls barrier properties, we have $h_{k}^{-}=h_{k}^{+}$if $k \neq c$.

Corollary 13. If $M$ and $N$ are compact metric spaces, $V: M \times$ $N \times M \times N \rightarrow \mathbb{R}$ is a Lipschitz function, and $(x, y) \in M \times N$, then

$$
\sup _{n \in \mathbb{Z}^{+}}|n c+h(n)(x, y)|<+\infty,
$$

where $c$ is the critical value.

Proof. There is $N \in \mathbb{Z}^{+}$such that

$$
h_{c}^{-}(x, y)-1<n c+h(n)(x, y)<h_{c}^{+}(x, y)+1,
$$


for $n \geq N$. Let $B$ be the maximum of numbers $\mid h_{c}^{-}(x, y)-$ $1|,| h_{c}^{+}(x, y)+1|| c+,h(1)(x, y)|| 2 c+,h(2)(x, y)|, \ldots|,(N-$ 1) $c+h(N-1)(x, y) \mid$. According to Theorems 2 and 12, we have $h_{c}^{-}(x, y), h_{c}^{+}(x, y) \in \mathbb{R}$, so $B \in \mathbb{R}$, and in addition,

$$
|n c+h(n)(x, y)| \leq B
$$

for all $n \in \mathbb{Z}^{+}$.

Corollary 14. If $M$ and $N$ are compact metric spaces, $V: M \times$ $N \times M \times N \rightarrow \mathbb{R}$ is a Lipschitz function, and $(x, y) \in M \times N$, then

$$
-c=\lim _{n \rightarrow \infty} \frac{1}{n} h(n)(x, y),
$$

where $c$ is the critical value.

Theorem 3 states that $c$ can be written in terms of actions for certain special sequences, using a solution of the Hamilton-Jacobi equation.

Proof. Consider $n \in \mathbb{Z}^{+},(x, y) \in M \times N$, and $u \in C(M \times N, \mathbb{R})$ solutions of the Hamilton-Jacobi equation (66). Because $M$ and $N$ are compact spaces, for each $n \in \mathbb{Z}^{+}$, given $x_{n} \in M$, we can choose

$$
\begin{array}{r}
y_{n}^{*}\left(x_{n}\right) \in \underset{y_{n} \in N}{\arg \min }\left\{u\left(x_{n}, y_{n}\right)-u\left(x_{n-1}^{*}, y_{n-1}^{*}\left(x_{n-1}^{*}\right)\right)\right. \\
\left.-V\left(x_{n-1}^{*}, y_{n-1}^{*}\left(x_{n-1}^{*}\right), x_{n}, y_{n}\right)\right\},
\end{array}
$$

where $\left(x_{0}^{*}, y_{0}^{*}\left(x_{0}^{*}\right)\right)=(x, y)$. We may now choose

$$
\begin{aligned}
x_{n}^{*} \in \underset{x_{n} \in M}{\arg \max }\{ & u\left(x_{n}, y_{n}^{*}\left(x_{n}\right)\right)-u\left(x_{n-1}^{*}, y_{n-1}^{*}\left(x_{n-1}^{*}\right)\right) \\
& \left.-V\left(x_{n-1}^{*}, y_{n-1}^{*}\left(x_{n-1}^{*}\right), x_{n}, y_{n}^{*}\left(x_{n}\right)\right)\right\} .
\end{aligned}
$$

On account of $u$ is the solution of (66), we have

$$
\begin{aligned}
c= & u\left(x_{n}^{*}, y_{n}^{*}\left(x_{n}^{*}\right)\right)-u\left(x_{n-1}^{*}, y_{n-1}^{*}\left(x_{n-1}^{*}\right)\right) \\
& -V\left(x_{n-1}^{*}, y_{n-1}^{*}\left(x_{n-1}^{*}\right), x_{n}^{*}, y_{n}^{*}\left(x_{n}^{*}\right)\right) .
\end{aligned}
$$

Consequently, for each $n \in \mathbb{Z}^{+}$, we have

$$
n c=u\left(x_{n}^{*}, y_{n}^{*}\left(x_{n}^{*}\right)\right)-u(x, y)-A^{n}\left(\bar{x}^{*}(n), \bar{y}^{*}(n)\right),
$$

where $\bar{x}^{*}(n)=\left\{x_{i}^{*}\right\}_{i=0}^{n} \in S_{M}(x, n)$ and $\bar{y}^{*}(n)=\left\{y_{i}^{*}\left(x_{i}^{*}\right)\right\}_{i=0}^{n} \in$ $S_{N}(y, n)$.

By the compactness of $M$ and $N$ and because $u \in C(M \times$ $N, \mathbb{R})$ we obtain that

$$
\inf u-\sup u \leq n c+A^{n}\left(\bar{x}^{*}(n), \bar{y}^{*}(n)\right) \leq \sup u-\inf u,
$$

from which we conclude that

$$
\left|n c+A^{n}\left(\bar{x}^{*}(n), \bar{y}^{*}(n)\right)\right| \leq \sup u-\inf u<+\infty,
$$

for all $n \in \mathbb{Z}^{+}$.
We can obtain another characterization of the critical value in terms of optimal sequences in the previous theorem.

Corollary 15. If $M$ and $N$ are compact metric spaces, $V: M \times$ $N \times M \times N \rightarrow \mathbb{R}$ is a Lipschitz function, and $(x, y) \in M \times N$, then there are sequences $\left\{x_{i}^{*}\right\}_{i=0}^{\infty} \subset M$ and $\left\{y_{i}^{*}\right\}_{i=0}^{\infty} \subset N$ such that

$$
-c=\lim _{n \rightarrow \infty} \frac{1}{n} A^{n}\left(\bar{x}^{*}(n), \bar{y}^{*}(n)\right)
$$

where $\bar{x}^{*}(n)=\left\{x_{i}^{*}\right\}_{i=0}^{n} \in S_{M}(x, n)$ and $\bar{y}^{*}(n)=\left\{y_{i}^{*}\right\}_{i=0}^{n} \in$ $S_{N}(y, n)$, where $c$ is the critical value.

\section{Critical Solutions}

Let $M$ and $N$ be compact metric spaces, and let $V: M \times N \times$ $M \times N \rightarrow \mathbb{R}$ be a Lipschitz function.

Definition 16. For $k \in \mathbb{R}$, a function $g \in C(M \times N, \mathbb{R})$ is called $k$-supersolution if

$$
\mathscr{L}^{1} g \geq g+k
$$

Analogously, a function $g \in C(M \times N, \mathbb{R})$ is called $k$-subsolution if

$$
\mathscr{L}^{1} g \leq g+k
$$

The function $g$ is a $k$-solution, if $g$ is $k$-supersolution and $k$ subsolution.

It is evident that $g$ is a $k$-solution if and only if $g$ is a fixed point of the Lax operator with critical value $k$; in this case $k=$ $c$ according to Theorem 1 . The $c$-solutions are called critical solutions.

Proposition 17. If $M$ and $N$ are compact metric spaces, $V$ : $M \times N \times M \times N \rightarrow \mathbb{R}$ is a Lipschitz function, $k \in \mathbb{R}$, and $\left(x_{0}, y_{0}\right) \in M \times N$, then

(1) $g$ is $k$-supersolution if and only if there exists $\widehat{x}_{1} \in M$ such that

$$
g\left(\widehat{x}_{1}, y_{1}\right)-g\left(x_{0}, y_{0}\right)-V\left(x_{0}, y_{0}, \widehat{x}_{1}, y_{1}\right) \geq k
$$

for all $y_{1} \in N$;

(2) $g$ is $k$-subsolution if and only if for every $x_{1} \in M$, there exists $\widehat{y}_{1}=\widehat{y}_{1}\left(x_{1}\right) \in N$ such that

$$
g\left(x_{1}, \widehat{y}_{1}\right)-g\left(x_{0}, y_{0}\right)-V\left(x_{0}, y_{0}, x_{1}, \widehat{y}_{1}\right) \leq k
$$

Proof. Consider $\left(x_{0}, y_{0}\right) \in M \times N$,

(1) $g$ satisfies (108) if and only if there is $\widehat{x}_{1} \in M$ such that

$$
\begin{gathered}
\inf _{y_{1} \in N}\left\{g\left(\widehat{x}_{1}, y_{1}\right)-V\left(x_{0}, y_{0}, \widehat{x}_{1}, y_{1}\right)\right\} \\
\geq g\left(x_{0}, y_{0}\right)+k,
\end{gathered}
$$


so

$$
\begin{aligned}
& \mathscr{L}^{1} g\left(x_{0}, y_{0}\right) \\
& =\inf _{\Theta(1)} \sup _{\bar{x}(1)}\left\{g\left(x_{1}, \Theta(1)[\bar{x}(1)]_{1}\right)\right. \\
& \left.\quad-V\left(x_{0}, y_{0}, x_{1}, \Theta(1)[\bar{x}(1)]_{1}\right)\right\} \\
& =\sup _{x_{1} \in M} \inf _{y_{1} \in N}\left\{g\left(x_{1}, y_{1}\right)-V\left(x_{0}, y_{0}, x_{1}, y_{1}\right)\right\} \\
& \geq g\left(x_{0}, y_{0}\right)+k,
\end{aligned}
$$

where $\bar{x}(1) \in S_{M}\left(x_{0}, 1\right)$ and $\Theta(1) \in \mathscr{E}_{N}\left(x_{0}, y_{0}, 1\right)$;

(2) $g$ satisfies (109) if and only if for every $x_{1} \in M$, we have

$$
\begin{gathered}
\inf _{y_{1} \in N}\left\{g\left(x_{1}, y_{1}\right)-V\left(x_{0}, y_{0}, x_{1}, y_{1}\right)\right\} \\
\leq g\left(x_{0}, y_{0}\right)+k .
\end{gathered}
$$

Next,

$$
\begin{aligned}
& \mathscr{L}^{1} g\left(x_{0}, y_{0}\right) \\
& =\inf _{\Theta(1) \bar{x}(1)}\left\{g\left(x_{1}, \Theta(1)[\bar{x}(1)]_{1}\right)\right. \\
& \left.\quad-V\left(x_{0}, y_{0}, x_{1}, \Theta(1)[\bar{x}(1)]_{1}\right)\right\} \\
& =\sup _{x_{1} \in M_{y_{1}} \in N}\left\{g\left(x_{1}, y_{1}\right)-V\left(x_{0}, y_{0}, x_{1}, y_{1}\right)\right\} \\
& \leq g\left(x_{0}, y_{0}\right)+k,
\end{aligned}
$$

where $\bar{x}(1) \in S_{M}\left(x_{0}, 1\right)$ and $\Theta(1) \in \mathscr{E}_{N}\left(x_{0}, y_{0}, 1\right)$.

We have another consequence of the previous result: if $n \in$ $\mathbb{Z}^{+}$, the critical solutions $u$ satisfy the following statements:

(1) for every strategy $\Theta(n) \in \mathscr{E}_{N}\left(x_{0}, y_{0}, n\right)$, there exists $\widehat{\bar{x}}(n) \in S_{M}\left(x_{0}, n\right)$ such that

$$
\begin{aligned}
& u\left(x_{n}, \Theta(n)[\hat{\bar{x}}(n)]_{n}\right) \\
& \quad-u\left(x_{0}, y_{0}\right)-A^{n}(\widehat{\bar{x}}(n), \Theta(n)[\hat{\bar{x}}(n)]) \geq n c ;
\end{aligned}
$$

(2) there exists a strategy $\widehat{\Theta}(n) \in \mathscr{E}_{N}\left(x_{0}, y_{0}, n\right)$ such that

$$
\begin{array}{r}
u\left(x_{n}, \widehat{\Theta}(n)[\bar{x}(n)]_{n}\right) \\
-u\left(x_{0}, y_{0}\right)-A^{n}(\bar{x}(n), \widehat{\Theta}(n)[\bar{x}(n)]) \leq n c, \\
\forall \bar{x}(n) \in S_{M}\left(x_{0}, n\right) .
\end{array}
$$

We have some properties of $k$-super and $k$-subsolutions.
Lemma 18. For $g \in C(M \times N, \mathbb{R})$ and $\left(x_{0}, y_{0}\right) \in M \times N$,

(1) if $g$ is $k$-supersolution and $k^{\prime} \leq k$, then $g$ is $k^{\prime}$-supersolution;

(2) if $g$ is $k$-subsolution and $k^{\prime} \geq k$, then $g$ is $k^{\prime}$-subsolution;

(3) if the sets $S_{U}, S_{L} \subset \mathbb{R}$ are defined as

$$
\begin{gathered}
S_{U}(g)=\{k \in \mathbb{R}: g \text { isk-supersolution }\}, \\
S_{L}(g)=\{k \in \mathbb{R}: g \text { is } k \text {-subsolution }\},
\end{gathered}
$$

then $\sup S_{U}(g) \leq \inf S_{L}(g)$.

Proof. The first and second items are clear. To prove the third claim, choose $a \in S_{U}(g)$ and $b \in S_{L}(g)$. Then, there is $\widehat{x}_{1} \in M$ such that

$$
g\left(\widehat{x}_{1}, y_{1}\right)-g\left(x_{0}, y_{0}\right)-V\left(x_{0}, y_{0}, \widehat{x}_{1}, y_{1}\right) \geq a,
$$

for all $y_{1} \in N$. Because $b \in S_{L}(g)$, there is $\widehat{y}_{1}=\widehat{y}_{1}\left(\widehat{x}_{1}\right)$ such that

$$
g\left(\widehat{x}_{1}, \hat{y}_{1}\right)-g\left(x_{0}, y_{0}\right)-V\left(x_{0}, y_{0}, \widehat{x}_{1}, \hat{y}_{1}\right) \leq b .
$$

Therefore, $a \leq b$, and then $\sup S_{U}(g) \leq \inf S_{L}(g)$.

By definition, if $u$ is a fixed point of the Lax operator $\mathscr{L}^{n}$ with critical value $c$, then $u$ is a critical solution. Therefore, $c \in S_{U}(u) \cap S_{L}(u)$, and according to Lemma 18 , we have a new characterization of the critical value $c$ (see Theorem 4).

We are interested in showing that the lower Peierls barrier is a critical solution. To this end, we consider the behavior of extremal sequences for lower Peierls barrier defined as follows.

Definition 19. We define a couple $(\widehat{\bar{x}}(n), \widehat{\Theta}(n)) \in S_{M}\left(x_{0}, n\right) \times$ $\mathscr{E}_{N}\left(x_{0}, y_{0}, n\right)$ extremal for $h(n)\left(x_{0}, y_{0}\right)$, if $h(n)\left(x_{0}, y_{0}\right)=$ $A^{n}(\hat{\bar{x}}(n), \widehat{\Theta}(n)[\hat{\bar{x}}(n)])$ and satisfies the following conditions:

(1) for every strategy $\Theta(n) \in \mathscr{E}_{N}\left(x_{0}, y_{0}, n\right)$, there exists $\widetilde{\bar{x}}(n) \in S_{M}\left(x_{0}, n\right)$ such that

$$
A^{n}(\widehat{\bar{x}}(n), \widehat{\Theta}(n)[\widehat{\bar{x}}(n)]) \geq A^{n}(\widetilde{\bar{x}}(n), \Theta(n)[\widetilde{\bar{x}}(n)])
$$

(2) for every $\bar{x}(n) \in S_{M}\left(x_{0}, n\right)$,

$$
A^{n}(\widehat{\bar{x}}(n), \widehat{\Theta}(n)[\widehat{\bar{x}}(n)]) \leq A^{n}(\bar{x}(n), \widehat{\Theta}(n)[\bar{x}(n)]) .
$$

The following result tells us that the lower Peierls barrier is a critical solution and therefore a solution of the HamiltonJacobi equation.

\section{Theorem 20. $-h_{c}^{-}$is a critical solution.}

Proof. Consider $\left(x_{0}, y_{0}\right) \in M \times N$. According to Theorem 2, $h_{c}^{-} \in \mathbb{R}$.

(I) We begin by proving $-h_{c}^{-}$is $c$-supersolution. Let us take $y_{1} \in N$ and let $\widehat{\Phi}(n-1) \in \mathscr{E}_{N}\left(x_{1}, y_{1}, n-1\right)$ be extremal for $h(n-1)\left(x_{1}, y_{1}\right)$, then 


$$
\begin{aligned}
& h(n-1)\left(x_{1}, y_{1}\right) \\
& \quad=\inf _{\bar{z}(n-1)} A^{n-1}(\bar{z}(n-1), \widehat{\Phi}(n-1)[\bar{z}(n-1)]),
\end{aligned}
$$

where $\bar{z}(n-1) \in S_{M}\left(x_{1}, n-1\right)$.

For every $\bar{x}(n) \in S_{M}\left(x_{0}, n\right)$, define $\widehat{\Theta}(n) \in \mathscr{E}_{N}\left(x_{0}, y_{0}, n\right)$ as

$$
\widehat{\Theta}(n)[\bar{x}(n)]_{i}= \begin{cases}y_{0}, & i=0, \\ \widehat{\Phi}(n-1)\left[\left\{x_{1}, x_{2}, \ldots, x_{n}\right\}\right]_{i-1}, & 1 \leq i \leq n,\end{cases}
$$

then

$$
h_{c}^{-}\left(x_{0}, y_{0}\right) \geq \liminf _{n \rightarrow \infty}\left\{\inf _{\bar{x}(n)} A^{n}(\bar{x}(n), \widehat{\Theta}(n)[\bar{x}(n)])+c n\right\} .
$$

There is $\widehat{\overline{\bar{x}}}(n) \in S_{M}\left(x_{0}, n\right)$ such that

$$
\inf _{\bar{x}(n)} A^{n}(\bar{x}(n), \widehat{\Theta}(n)[\bar{x}(n)])=A^{n}(\hat{\bar{x}}(n), \widehat{\Theta}(n)[\hat{\bar{x}}(n)]) .
$$

Choose a subsequence $n_{j} \uparrow \infty$ such that $n_{1}=1$ and

$$
\begin{aligned}
& \liminf _{n \rightarrow \infty} A^{n}(\widehat{\bar{x}}(n), \widehat{\Theta}(n)[\hat{\bar{x}}(n)])+n c \\
& \quad=\lim _{j \rightarrow \infty} A^{n_{j}}\left(\hat{\bar{x}}\left(n_{j}\right), \widehat{\Theta}\left(n_{j}\right)\left[\hat{\bar{x}}\left(n_{j}\right)\right]\right)+n_{j} c ;
\end{aligned}
$$

extracting a subsequence if necessary, we can assume that

$$
\widehat{\bar{x}}\left(n_{j}\right) \longrightarrow \bar{x}^{*} \in S_{M}\left(x_{0}, \infty\right),
$$

where $S_{M}\left(x_{0}, \infty\right)$ denotes the set of infinite sequences in $M$, with initial condition $x_{0}$. Therefore,

$$
\begin{aligned}
& h_{c}^{-}\left(x_{0}, y_{0}\right) \\
& \geq \lim _{j \rightarrow \infty}\left\{V\left(x_{0}, y_{0}, x_{1}^{*}, y_{1}\right)+c\right. \\
& +\left[V\left(x_{0}, y_{0}, \widehat{\bar{x}}\left(n_{j}\right)_{1}, \widehat{\Theta}\left(n_{j}\right)\left[\widehat{\bar{x}}\left(n_{j}\right)\right]_{1}\right)\right. \\
& \left.-V\left(x_{0}, y_{0}, x_{1}^{*}, y_{1}\right)\right] \\
& +\left[V \left(\widehat{\bar{x}}\left(n_{j}\right)_{1}, \widehat{\Theta}\left(n_{j}\right)\left[\widehat{\bar{x}}\left(n_{j}\right)\right]_{1},\right.\right. \\
& \left.\widehat{\bar{x}}\left(n_{j}\right)_{2}, \widehat{\Theta}\left(n_{j}\right)\left[\widehat{\bar{x}}\left(n_{j}\right)\right]_{2}\right) \\
& \left.-V\left(x_{1}^{*}, y_{1}, \widehat{\bar{x}}\left(n_{j}\right)_{2}, \widehat{\Theta}\left(n_{j}\right)\left[\widehat{\bar{x}}\left(n_{j}\right)\right]_{2}\right)\right] \\
& +A^{n_{j}-1}\left(\widehat{\bar{z}}\left(n_{j}-1\right), \widehat{\Phi}\left(n_{j}-1\right)\left[\widehat{\bar{z}}\left(n_{j}-1\right)\right]\right) \\
& \left.+\left(n_{j}-1\right) c\right\}
\end{aligned}
$$

where $\widehat{\bar{z}}\left(n_{j}-1\right)=\left\{x_{1}^{*}, \widehat{x}_{2}, \ldots, \widehat{x}_{n_{j}}\right\}$. Because $V$ is continuous, then

$$
\begin{aligned}
& h_{c}^{-}\left(x_{0}, y_{0}\right) \\
& \geq V\left(x_{0}, y_{0}, x_{1}^{*}, y_{1}\right)+c \\
& +\liminf _{n \rightarrow \infty}\left\{\inf _{\bar{z}(n-1)} A^{n-1}(\widehat{\bar{z}}(n-1),\right. \\
& \quad \widehat{\Phi}(n-1)[\widehat{\bar{z}}(n-1)]) \\
& +(n-1) c\}
\end{aligned}
$$$$
=V\left(x_{0}, y_{0}, x_{1}^{*}, y_{1}\right)+c+h_{c}^{-}\left(x_{1}^{*}, y_{1}\right) \text {, }
$$

which proves that there is $x_{1}^{*} \in M$ such that

$$
-h_{c}^{-}\left(x_{1}^{*}, y_{1}\right)+h_{c}^{-}\left(x_{0}, y_{0}\right)-V\left(x_{0}, y_{0}, x_{1}^{*}, y_{1}\right) \geq c,
$$

for all $y_{1} \in N$. Hence, $-h_{c}^{-}$is $c$-supersolution.

(II) We proceed to show that $-h_{c}^{-}$is $c$-subsolution. Let us take $x_{1} \in M$. For each $n \in \mathbb{Z}^{+}$, let $\widehat{\Theta}(n) \in \mathscr{E}_{N}\left(x_{0}, y_{0}, n\right)$ be extremal for $h(n)\left(x_{0}, y_{0}\right)$, then

$$
h(n)\left(x_{0}, y_{0}\right)=\inf _{\bar{x}(n)} A^{n}(\bar{x}(n), \widehat{\Theta}(n)[\bar{x}(n)]) .
$$

Observe that $\widehat{\Theta}(n)[\bar{x}(n)]_{1}$ depends only on $x_{1}$, because $\widehat{\Theta}(n) \epsilon$ $\mathscr{E}_{N}\left(x_{0}, y_{0}, n\right)$. Suppose that $\widehat{\Theta}(n)[\bar{x}(n)]_{1} \rightarrow y_{1}^{*}\left(x_{1}\right)$.

For $\bar{w}(n-1)=\left\{x_{1}, w_{2}, \ldots, w_{n}\right\} \in S_{M}\left(x_{1}, n-1\right)$, we can define a strategy $\widehat{\Phi}(n-1) \in \mathscr{E}_{N}\left(x_{1}, y_{1}^{*}\left(x_{1}\right), n-1\right)$ as follows:

$$
\widehat{\Phi}(n-1)[\bar{w}(n-1)]_{i}= \begin{cases}y_{1}^{*}\left(x_{1}\right), & i=0, \\ \widehat{\Theta}(n)[\bar{z}(n)]_{i+1}, & 1 \leq i \leq n-1,\end{cases}
$$

where $\bar{z}(n)=\left\{x_{0}, x_{1}, w_{2}, w_{3}, \ldots, w_{n}\right\} \in S_{M}\left(x_{0}, n\right)$. So

$$
\begin{aligned}
& h(n-1)\left(x_{1}, y_{1}^{*}\left(x_{1}\right)\right) \\
& \quad \geq \inf _{\bar{w}(n-1)} A^{n-1}(\bar{w}(n-1), \widehat{\Phi}(n-1)[\bar{w}(n-1)]) .
\end{aligned}
$$

We can choose $\widehat{\bar{w}}(n-1)=\left\{x_{1}, \widehat{w}_{2}, \ldots, \widehat{w}_{n}\right\} \in S_{M}\left(x_{1}, n-1\right)$ extremal for

$$
\inf _{\bar{w}(n-1)} A^{n-1}(\bar{w}(n-1), \widehat{\Phi}(n-1)[\bar{w}(n-1)]),
$$

and a subsequence $n_{j} \uparrow \infty$ such that

$$
\begin{aligned}
& h_{c}^{-}\left(x_{1}, y_{1}^{*}\left(x_{1}\right)\right) \\
& \geq \lim _{j \rightarrow \infty} A^{n_{j}-1}\left(\widehat{\bar{w}}\left(n_{j}-1\right), \widehat{\Phi}\left(n_{j}-1\right)\left[\widehat{\bar{w}}\left(n_{j}-1\right)\right]\right) \\
& \quad+c\left(n_{j}-1\right) .
\end{aligned}
$$




$$
\begin{aligned}
& \text { If } \widehat{\bar{z}}\left(n_{j}\right)=\left\{x_{0}, x_{1}, \widehat{w}_{2}, \widehat{w}_{3}, \ldots, \widehat{w}_{n_{j}}\right\} \text {, then } \\
& h_{c}^{-}\left(x_{0}, y_{0}\right) \\
& =\operatorname{liminfinf}_{n \rightarrow \infty} A_{\bar{x}(n)}^{n}(\bar{x}(n), \widehat{\Theta}(n)[\bar{x}(n)])+n c \\
& \leq \lim _{j \rightarrow \infty}\left\{V\left(x_{0}, y_{0}, x_{1}, y_{1}^{*}\left(x_{1}\right)\right)+c\right. \\
& +\left[V\left(x_{0}, y_{0}, x_{1}, \widehat{\Theta}\left(n_{j}\right)\left[\widehat{\bar{z}}\left(n_{j}\right)\right]_{1}\right)\right. \\
& \left.-V\left(x_{0}, y_{0}, x_{1}, y_{1}^{*}\left(x_{1}\right)\right)\right] \\
& +\left[V \left(x_{1}, \widehat{\Theta}\left(n_{j}\right)\left[\widehat{\bar{z}}\left(n_{j}\right)\right]_{1},\right.\right. \\
& \left.\widehat{\bar{z}}\left(n_{j}\right)_{2}, \widehat{\Theta}\left(n_{j}\right)\left[\widehat{\bar{z}}\left(n_{j}\right)\right]_{2}\right) \\
& -V\left(x_{1}, y_{1}^{*}\left(x_{1}\right), \hat{\bar{z}}\left(n_{j}\right)_{2}\right. \text {, } \\
& \left.\left.\widehat{\Theta}\left(n_{j}\right)\left[\widehat{\bar{z}}\left(n_{j}\right)\right]_{2}\right)\right] \\
& +A^{n_{j}-1}\left(\widehat{\bar{w}}\left(n_{j}-1\right),\right. \\
& \left.\widehat{\Phi}\left(n_{j}-1\right)\left[\widehat{\bar{w}}\left(n_{j}-1\right)\right]\right) \\
& \left.+\left(n_{j}-1\right) c\right\} \text {. }
\end{aligned}
$$

According to the above relations and the continuity of $V$, if $x_{1} \in M$, there is $y_{1}^{*}\left(x_{1}\right) \in N$ such that

$$
\begin{array}{r}
-h_{c}^{-}\left(x_{1}, y_{1}^{*}\left(x_{1}\right)\right)+h_{c}^{-}\left(x_{0}, y_{0}\right) \\
-V\left(x_{0}, y_{0}, x_{1}, y_{1}^{*}\left(x_{1}\right)\right) \leq c .
\end{array}
$$

Therefore, $-h_{c}^{-}$is a $c$-subsolution.

\section{References}

[1] A. Fathi, "Théorème KAM faible et théorie de Mather sur les systèmes lagrangiens," Comptes Rendus de l'Académie des Sciences, vol. 324, no. 9, pp. 1043-1046, 1997.

[2] A. Fathi, "Sur la convergence du semi-groupe de Lax-Oleinik," Comptes Rendus de l'Académie des Sciences, vol. 327, no. 3, pp. 267-270, 1998.

[3] A. Fathi, Weak KAM Theorem in Lagrangian Dynamics, Preliminary Version, 2008.

[4] A. Fathi and A. Siconolfi, "Existence of $C^{1}$ critical subsolutions of the Hamilton-Jacobi equation," Inventiones Mathematicae, vol. 155, no. 2, pp. 363-388, 2004.

[5] R. Mañé, "Global variational methods in conservative dynamics," in 18 Colóquio Brasileiro de Matemática, Instituto de Matemática Pura e Aplicada (IMPA), Rio de Janeiro, Brazil, 1993.

[6] R. Mañé, "Lagrangian flows: the dynamics of globally minimizing orbits," Boletim da Sociedade Brasileira de Matemática, vol. 28, no. 2, pp. 141-153, 1997.

[7] J. N. Mather, "Action minimizing invariant measures for positive definite Lagrangian systems," Mathematische Zeitschrift, vol. 207, no. 2, pp. 169-207, 1991.
[8] W. H. Fleming and H. M. Soner, Controlled Markov Processes and Viscosity Solutions, vol. 25, Springer, New York, NY, USA, 2nd edition, 2006.

[9] M. Bardi and I. Capuzzo-Dolcetta, Optimal Control and Viscosity Solutions of Hamilton-Jacobi-Bellman Equations, Birkhäuser, Boston, Mass, USA, 1997.

[10] L. C. Evans and P. E. Souganidis, "Differential games and representation formulas for solutions of Hamilton-Jacobi-Isaacs equations," Indiana University Mathematics Journal, vol. 33, no. 5, pp. 773-797, 1984.

[11] D. A. Gomes, "Viscosity solution methods and the discrete Aubry-Mather problem," Discrete and Continuous Dynamical Systems, vol. 13, no. 1, pp. 103-116, 2005.

[12] G. Contreras and R. Iturriaga, Global Minimizers of Autonomous Lagrangians, Instituto de Matemática Pura e Aplicada (IMPA), Rio de Janeiro, Brazil, 1999.

[13] G. Contreras, "Action potential and weak KAM solutions," Calculus of Variations and Partial Differential Equations, vol. 13, no. 4, pp. 427-458, 2001. 


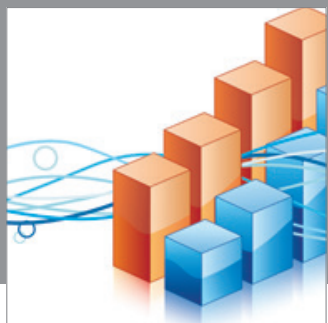

Advances in

Operations Research

mansans

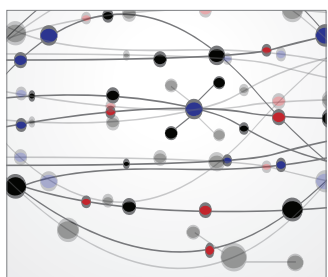

The Scientific World Journal
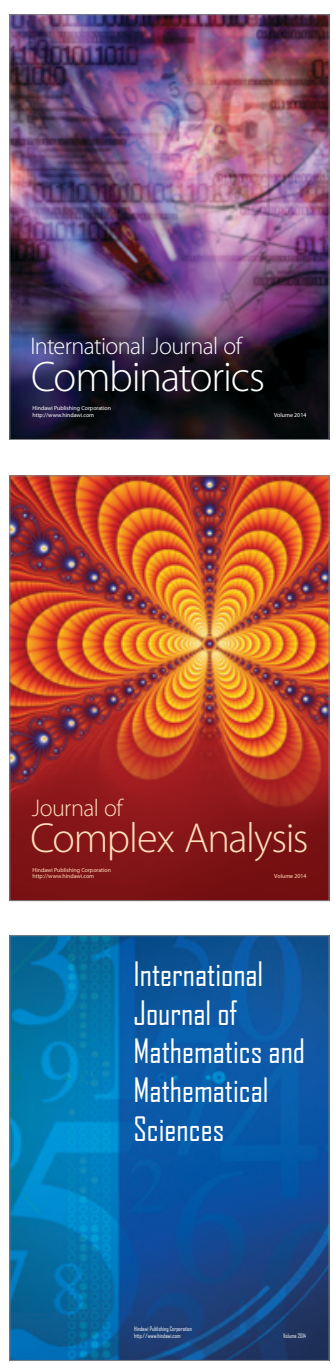
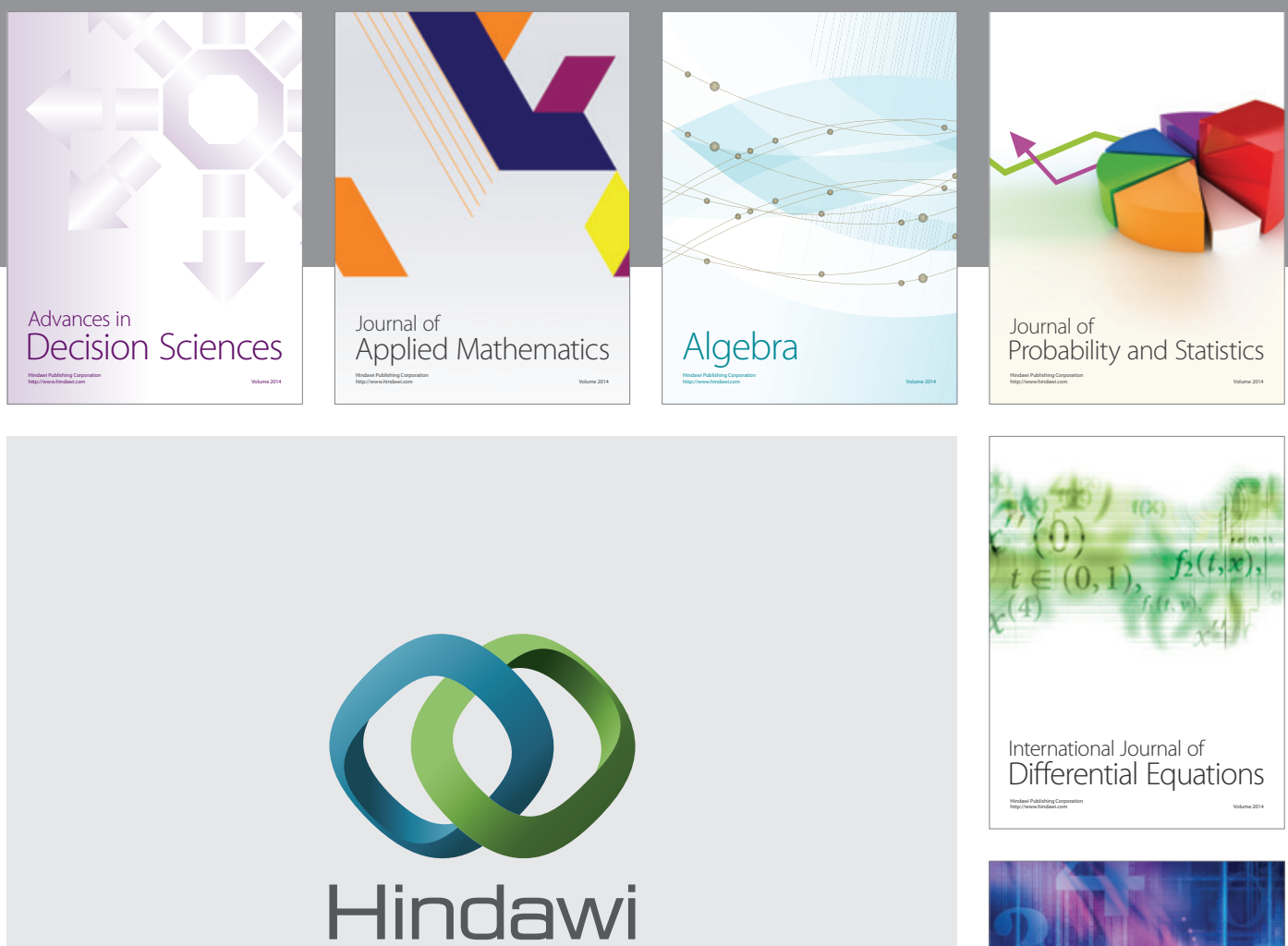

Submit your manuscripts at http://www.hindawi.com
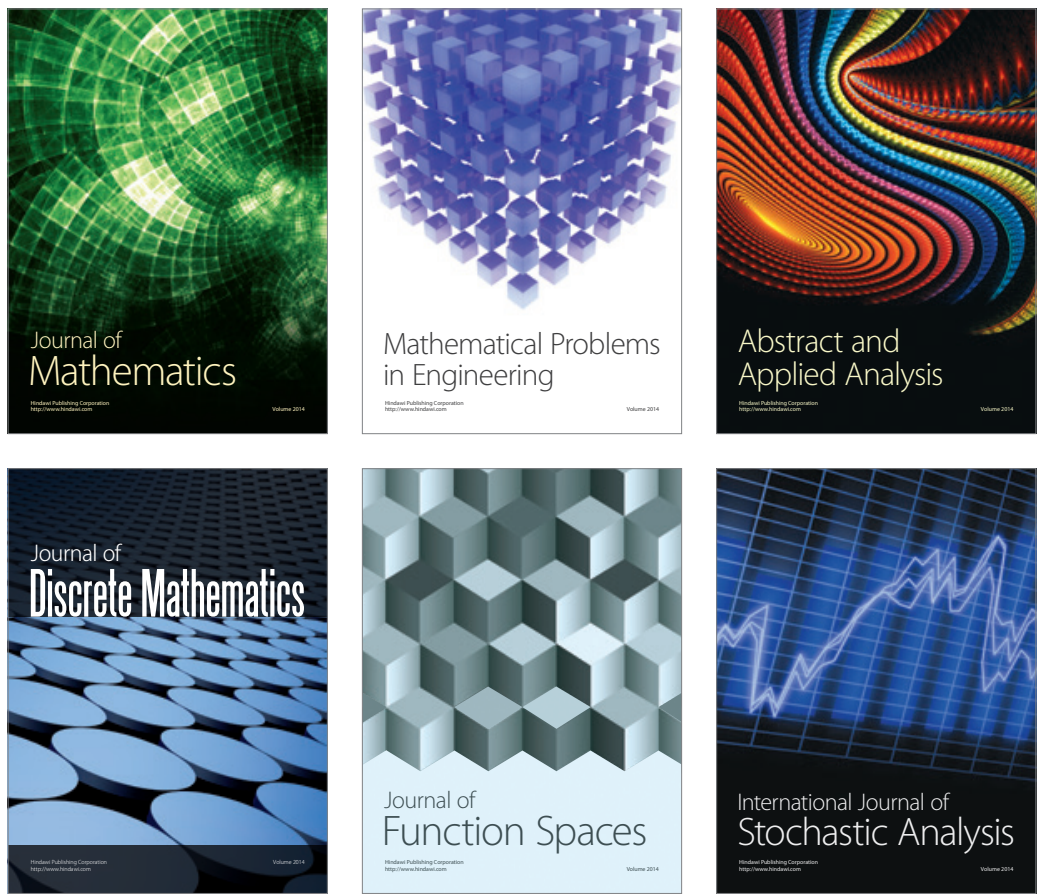

Journal of

Function Spaces

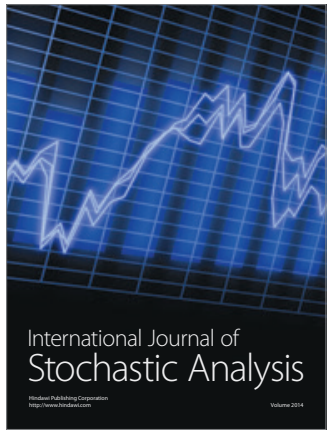

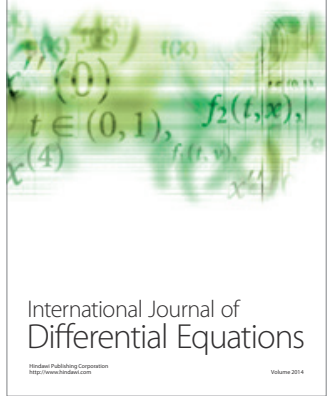
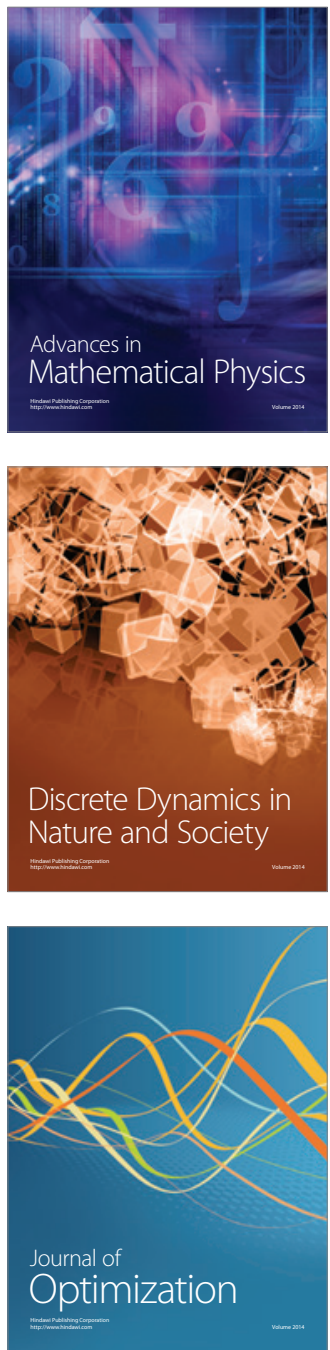